\title{
Numerical approach of determining the Stress Intensity Factor (KI) for a curved crack problem using Extended Isogeometric Analysis
}

\author{
SAID ELFAKKOUSS ${ }^{1}$, HASSANE MOUSTABCHIR $^{2}$, AHMED ELKHALFI $^{3}$ \\ \{said.elfakkoussi@gmail.com ${ }^{1}$, hmoustabchir@hotmail.com², aelkhalfi@gmail.com ${ }^{3}$ \} \\ Faculty of Science and Technology, Sidi Mohamed Ben Abdellah University Fez. Morocco ${ }^{1,3}$, \\ Laboratory of Systems Engineering and Applications (LISA), National School of Applied Sciences of \\ Fez. Sidi Mohamed Ben Abdellah University Fez. Morocco².
}

\begin{abstract}
The fracture of cracked metal structures remains the main concern of engineers and researchers in various fields (automotive, oil, naval and aeronautics, etc.). They are always looking for ways and tools to locate and determine where and when the break occurs. In the industrial field there are many instruments and devices for measuring and locating defects in metal structures. Prevention tools, however, remain the only way to understand or limit the source of the problem. The most efficient of these tools is the computer which helps to model the fracture phenomenon of cracked metal structures. In this work, we will study the problem of cracking in pipes using new interpolations functions called NURBS (rational non-uniform B-Splines). These functions are added to the formulation of the X-FEM method to study a 2D problem in the linear elastic domain. The geometry studied is a half-tube with a semi-elliptic crack. It should be kept in mind that the main purpose of this study is to calculate the KI factor with good precision, which will be used in determining the rate of rupture.
\end{abstract}

Keywords: Finite element method (FEM), Extended finite element method (X-FEM), Extended Isogeometric analysis (X-IGA), Semi-elliptical crack, B-Spline, NURBS Function, Interaction integral, Subroutine UEL, the Stress Intensity Factor (KI).

\section{Introduction}

The stress intensity factor $\mathrm{KI}$ is a parameter of linear elastic mechanics of rupture. It is used in particular to determine the degree of damage of cracked structures. Several methods can be used to determine this factor, namely, analytical, semi-analytical and numerical (FEM and XFEM). Numerical methods remain the most used tools in this field because of thier efficiency in terms of convergence, computation time and the actual modeling of the problem. The convergence of numerical methods is based on computer tools such as the power of machine servers, solvers, and the reliability of the calculation software used as well as the choice of programming language. To apply these tools in the industrial environment, especially to study the fracture of cracked pipelines, we will compute the KI factor numerically for a semi-elliptic crack in a half-tube (2D) by a new method named X-IGA implemented in the Abaqus / Standard software via the Subroutine UEL, based on the FORTRAN language [1]. El Fakkoussi et al. [1] already applied this implantation for a longitudinal crack plane in a half-tube (2D). It should be noted that the IGA and X-IGA approaches are only implemented in the Matlab software. So far, to our knowledge, this method has been partially implemented in the Abaqus / Standard software 
only on structures without cracks. This is why we propose to apply the new approach extended isogeometric analysis to the evaluation of the structure containing a crack and to present a special case to calculate the stress intensity factor (KI) using the approach extended isogeometric analysis for a pipeline containing an external semi-elliptic longitudinal plane crack. Finally, to prove the efficiency of the new strategy, we carried out a comparative study with analytical results witch exists in the literature and numerical extended finite element method (X-FEM).

\section{Theoretical Background.}

In this section, we will introduce the context of the IGA isogeometric analysis as well as the analytical solution for 2D half-pipe.

\subsection{Isogeometric analysis (IGA)}

B-Spline function. The B-Spline functions are written in the form of Cox-de Boor recursion formula [2]:

For $\mathrm{p}=0$

$$
N_{i, 0}(\xi)=\left\{\begin{array}{lr}
1 & \xi_{\mathrm{i}} \leq \xi \leq \xi_{\mathrm{i}+1} \\
0 & \text { otherwise }
\end{array}\right.
$$

For $p \geq 1$

$$
\mathrm{N}_{\mathrm{i}, \mathrm{p}}(\xi)=\frac{\xi-\xi_{\mathrm{i}}}{\xi_{\mathrm{i}+\mathrm{p}}-\xi_{\mathrm{i}}} \mathrm{N}_{\mathrm{i}, \mathrm{p}-1}(\xi)+\frac{\xi_{\mathrm{i}+\mathrm{p}+1}-\xi}{\xi_{\mathrm{i}+\mathrm{p}+1}-\xi_{\mathrm{i}+1}} \mathrm{~N}_{\mathrm{i}+1, \mathrm{p}-1}(\xi)
$$

The derivative of the B-Spline function is written as follows:

$$
\frac{\mathrm{dN}_{\mathrm{i}, \mathrm{p}}(\xi)}{\mathrm{d} \xi}=\frac{\mathrm{p}}{\xi_{\mathrm{i}+\mathrm{p}}-\xi_{\mathrm{i}}} \mathrm{N}_{\mathrm{i}, \mathrm{p}-1}(\xi)-\frac{\mathrm{p}}{\xi_{\mathrm{i}+\mathrm{p}+1}-\xi_{\mathrm{i}+1}} \mathrm{~N}_{\mathrm{i}+1, \mathrm{p}-1}(\xi)
$$

Non-Uniform Function Rational B-Spline (NURBS). These functions are a generalization of B-Spline functions based on rational functions: the NURBS functions are written as a fraction of two polynomials with real coefficients, in particular the B-Spline functions. These are identical to those used in CAD (computer-aided design). Indeed, these functions check the interpolation conditions [2]. It is important to point out that these functions were used in the isogeometric approach proposed in 2005 by Hughes [2]. 


$$
\begin{gathered}
\mathrm{R}_{\mathrm{i}, \mathrm{p}}(\xi)=\frac{\omega_{\mathrm{A}} \cdot N_{\mathrm{i}, \mathrm{p}}(\xi)}{\sum_{\mathrm{B}=1}^{\mathrm{n}} \omega_{A} \cdot N_{\mathrm{B}, \mathrm{p}}(\xi)} \\
\mathrm{R}_{\mathrm{A}}^{\mathrm{p}, \mathrm{q}}(\xi, \eta)=\frac{\omega_{A} \cdot N_{\mathrm{i}, \mathrm{p}}(\xi) \cdot \mathrm{M}_{\mathrm{i}, \mathrm{q}}(\eta)}{\sum_{\mathrm{i}=1}^{n} \sum_{\mathrm{j}=1}^{m} \omega_{A} \cdot N_{\mathrm{i}, \mathrm{p}}(\xi) \cdot \mathrm{M}_{\mathrm{j}, \mathrm{q}}(\eta)}
\end{gathered}
$$

With:

$\mathrm{N}_{\mathrm{i}, \mathrm{p}}(\xi)$ and $\mathrm{M}_{\mathrm{j}, \mathrm{q}}(\eta)$ are the basic functions B-Splines with $\mathrm{p}$ and $\mathrm{q}$ are the order of the interpolations function, $\mathrm{n}$ and $\mathrm{m}$ are the number of control points and are the weights corresponding to the control points $T=\left\{\zeta_{1}, \zeta_{2}, \ldots, \zeta_{n+p+1}\right\}$ and $H=\left\{\eta_{1}, \eta_{2}, \ldots, \eta_{n+q+1}\right\}$ are knots vectors (nodes).

Surface NURBS. To create B-Spline surfaces, it is necessary to build two-dimensional (2D) BSpline functions. To achieve this, it is sufficeint to use the tensor product which simply consists of multiplying the B-Spline functions into a single dimension (1D) in the two directions of space. The surface NURBS functions are represented by the following equation:

$$
S(\zeta, \eta)=\sum_{A=1}^{n . m} P_{A} \cdot R_{A}^{p, q}(\xi, \eta)
$$

With:

$P_{A}$ Is the control points, $R_{A}^{p, q}(\xi, \eta)$ is the bivariate NURBS basic functions.

\section{IGA / X-FEM implementation in Abaqus/Standard software}

The present work will apply this strategy to the implementation of the isogeometric analysis approach associated with the X-FEM method in the Abaqus / Standard software to study curved cracks in pipelines. Subsequently, the NURBS functions will be implemented in the Abaqus / Standard software via the User defined elements (UEL) subroutine which allows the finite element formulation in this software to be modified. Some other parameters of the XFEM method have been added in this code, such as the enrichment functions at the front and at the tip of the crack and the Heaviside function. the code UEL operation principle is detailed in figure 2. To make it easier to add additional information to the UEL (User defined elements), we have used the subroutine UEXTERNALDB (User-Defined External Databases). This will subsequently allow the addition of the necessary information on the crack and the NURBS function (figure1). These parameters are then used in the main program UEL (User defined elements). We find that El Fakkoussi et al. [1] have continued the work of Duval et al. [3] , whose goal is to couple the isogeometric analysis approach with the Extended Finite Element Method (X-FEM) to study plane cracking in pipelines. 


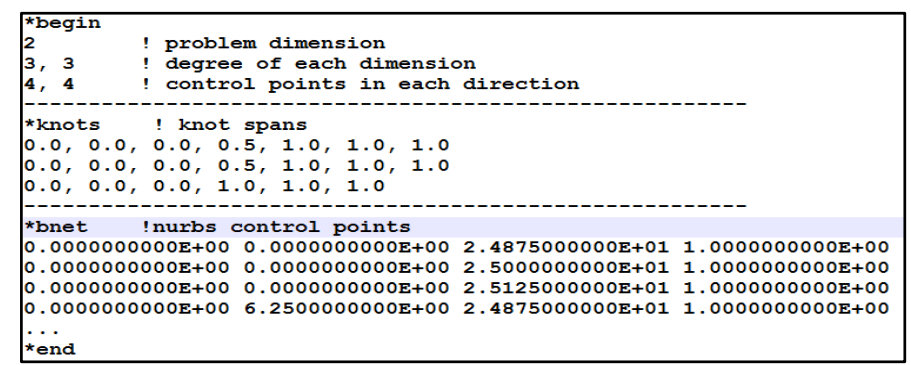

Fig. 1.Input file NURBS

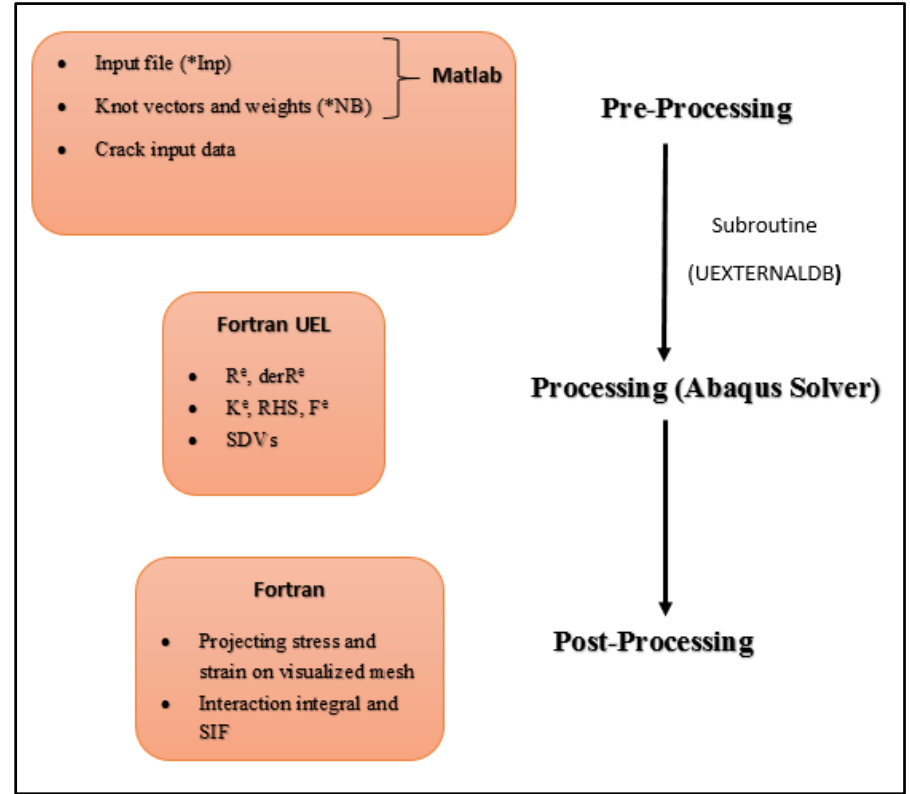

Fig .2. Implementation methodology of X-IGA approach in Abaqus/Standard software [1].

The calculation of results such as stresses and deformations determined by the extended isogeometric analysis (X-IGA) approach is based on the Bézier composition technique, which has been detailed in the references [4]. The extraction of the results and the main variables of the fracture mechanics are determined using the Fortran algorithm. This will help us program equation (7) to calculate, in a later step, the KI Constraint Intensity Factor as shown in Equation (10). 


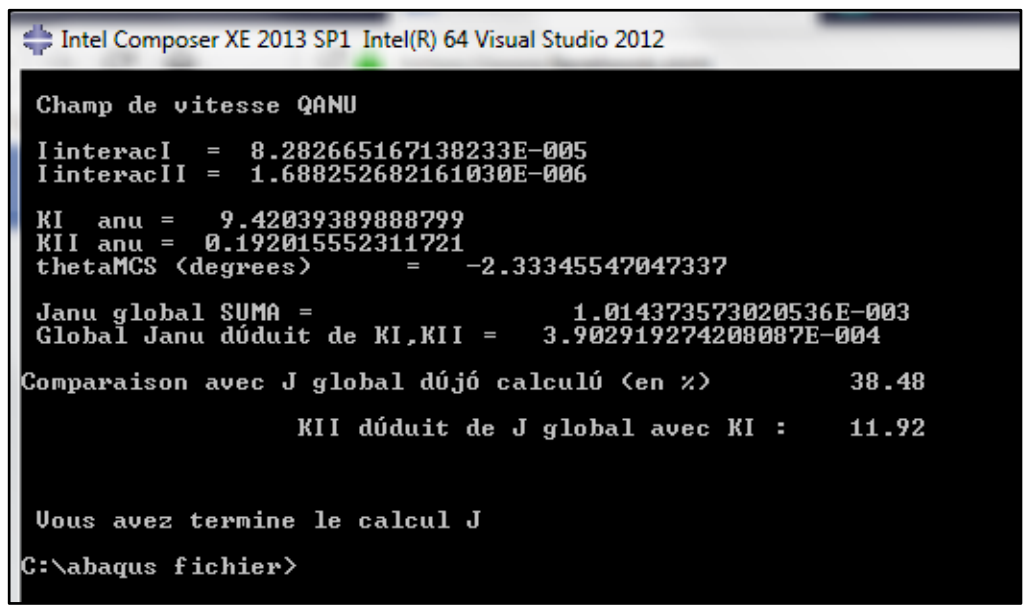

Fig. 3. FORTRAN algorithm for calculation the factor KI.

\section{Calculation of the Stress Intensity Factor (KI) for the curved crack}

The computation of the Stress Intensity Factor (KI) is based on the integral interaction method [5]. The mathematical formulation of this method for a two-dimensional problem is determined in the following way:

$$
M^{(1,2)}=\int_{A}\left[\sigma^{(1)}{ }_{i j} \frac{\delta u^{(2)}}{\delta x_{1}}+\sigma^{(2)}{ }_{i j} \frac{\delta u^{(1)}}{\delta x_{1}}-W^{(1,2)} \delta_{i j}\right] \frac{\delta q}{\delta x_{j}} d A
$$

With:

$q$ : Weight function

$q=1:$ In the crack tip and $q=0$ in the contour around the crack

$$
\begin{gathered}
W^{(1,2)}=\frac{1}{2}\left(\sigma^{(1)} \varepsilon^{(2)}+\sigma^{(2)} \varepsilon_{i j}^{(1)}{ }_{i j}\right)=\sigma^{(1)} \varepsilon_{i j}^{(2)}{ }_{i j}=\sigma^{(2)} \varepsilon^{(1)}{ }_{i j} \\
M^{(1,2)}=\frac{2}{\left(\frac{E}{1-\mu^{2}}\right)}\left(K^{(1)} K^{(2)}{ }_{I}+K^{(1)}{ }_{\|} K^{(2)}{ }_{I I}\right)
\end{gathered}
$$


For mode I, we have $\mathrm{K}^{(2)}{ }_{1}=1$ and $\mathrm{K}^{(2)}{ }_{\|}=0$

Finally, we get:

$$
\mathrm{K}_{\mathrm{I}}^{(1)}=\frac{\mathrm{M}^{(1, \mathrm{I})} \cdot\left(\frac{\mathrm{E}}{1-\mu^{2}}\right)}{2}
$$

The theoretical Stress Intensity Factor (KI) for mode I, in the case of a two-dimensional plate with a curved crack is written as follows [6]:

$$
\mathrm{K}_{\mathrm{l}}=\mathrm{F}_{1} \sigma \sqrt{\pi \mathrm{C}}
$$

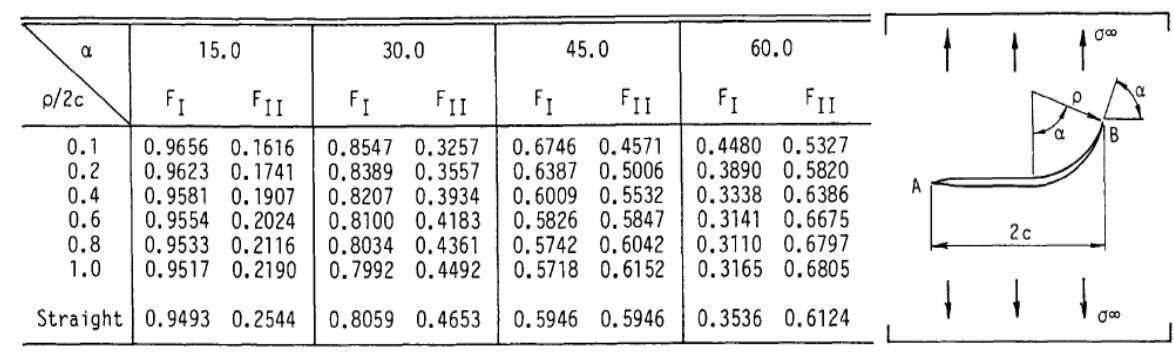

Fig. 4. Dimensionless KI of a crack that consists of a circular-arc part [6].

\section{$5 \quad$ Numerical simulation}

Numerical simulation is an important phase in the mechanical study of cracked pipelines, notably with the new X-IGA approach implemented in abaqus / standard software. To achieve this, we have used a half-tube (2D) with curved crack characterized by an inner radius and outer radius $\mathrm{Ri}=10 \mathrm{~mm}, \mathrm{Re}=20 \mathrm{~mm}$ and a crack length $\mathrm{a}=5 \mathrm{~mm}$ was used. The major details of a tube containing a curved crack and its boundary conditions were introduced in figure 5. The model studied is a static elastic linear, where the objective was to extract the values of the Stress Intensity Factor (KI). The Non-Uniform Functions Rational B-Spline used in this study are of the cubic order $(\mathrm{p}=\mathrm{q}=3)$. The number of elements used to mesh the twodimensional half-tube with curved crack are respectively 121 and 441 (figure 6). The material used in this study is generally used in gas and oil transport pipelines. the mechanical characteristics are shown in table 2 [7]: 
Table 1 : Mechanical properties of the material [7].

\begin{tabular}{|l|l|}
\hline Module d'Young & $\mathrm{E}=207000[\mathrm{MPa}]$ \\
\hline Poisson coefficient & $\mathrm{V}=0.3$ \\
\hline Yield strength & $\mathrm{Re}=340[\mathrm{MPa}]$ \\
\hline Ultimate tensile strength & $\mathrm{Rm}=440[\mathrm{MPa}]$ \\
\hline Elongation at break & $\mathrm{A}=35 \%$ \\
\hline
\end{tabular}

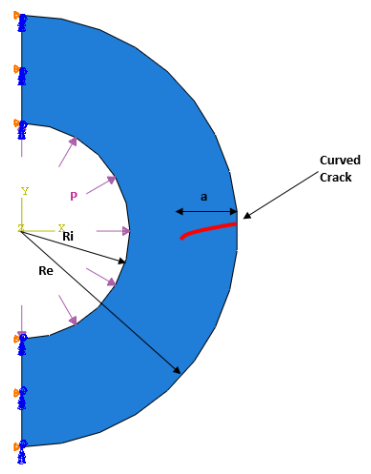

Fig.5. Annular tube with an axial crack and its boundary conditions.

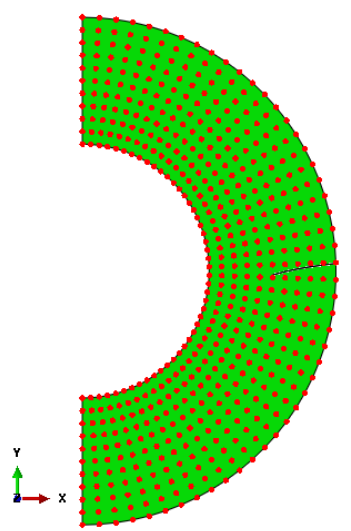

Fig. 6. Control point distribution for the X-IGA half-tube mesh with elliptic type crack. 


\section{$6 \quad$ Results and discussion}

Numerical simulation was done in the Abaqus / Standard software to implement the new Extended Isogeometric Analysis (X-IGA) approach to study curved cracks. We did the post-processing of the results in the Abaqus / Standard software using a program written in Fortran language. This program calculates the stress intensity factor KI Equation (10) based on integral interaction method the Equation (7). We used three half-tubes, two dimensional with three different cases of the mesh, a mesh of 121, 441 and 691 quadrilateral elements. Then, we compared the results arrived at with the analytical methods, the finite elements FEM and the method extended finite element method (X-FEM). The obtained results are shown in Table 2 . These results show a very good agreement with the other numerical results of the FEM method and the X-FEM method which do not exceed an error margin of $1.47 \%$. This means that the implementation of the new approach was successfull.

Table 2 : Comparison of KI factors between the proposed method X-IGA and the analytical, FEM and $\mathrm{X}$-FEM methods for the case of a crack with $\mathrm{a}=5 \mathrm{~mm}$.

\begin{tabular}{|c|c|c|c|c|}
\hline \multicolumn{5}{|c|}{ KI [MPa. $\left.\mathrm{mm}^{0.5}\right]$} \\
\hline & & & $X-I G A$ & $X-I G A$ \\
\hline Analytique [6] & FEM Method & X-FEM Method & 121 éléments & 441 éléments \\
\hline 4.75 & 4.68 & 4.71 & 4.7209 & 4.7269 \\
\hline Error (\%) & 1.47 & 0.84 & 0.61 & 0.48 \\
\hline
\end{tabular}

Figure 7 presents the comparison of the Von Mises constraints for the finite element method (FEM) and the extended finite element method (X-FEM) with the new Extended isogeometric analysis approach implemented in the Abaqus / Standard software. We used in this comparison a two-dimensional half-tube with an elliptical crack located in the outer surface of the tube under internal pressure, the study was carried out in the linear elastic domain. The results obtained after the simulation show a good agreement between the X-FEM method and the new X-IGA approach. The difference is due to the singularity problem near the tip of the crack whereas the FEM method is unable to capture the stresses near the crack front.

Simultaneously, we found that the constraints of Von Mises for the X-IGA method are close to the X-FEM method because of the enrichment functions already added to avoid the problem of the singularity of the close stresses at the crack tip. 

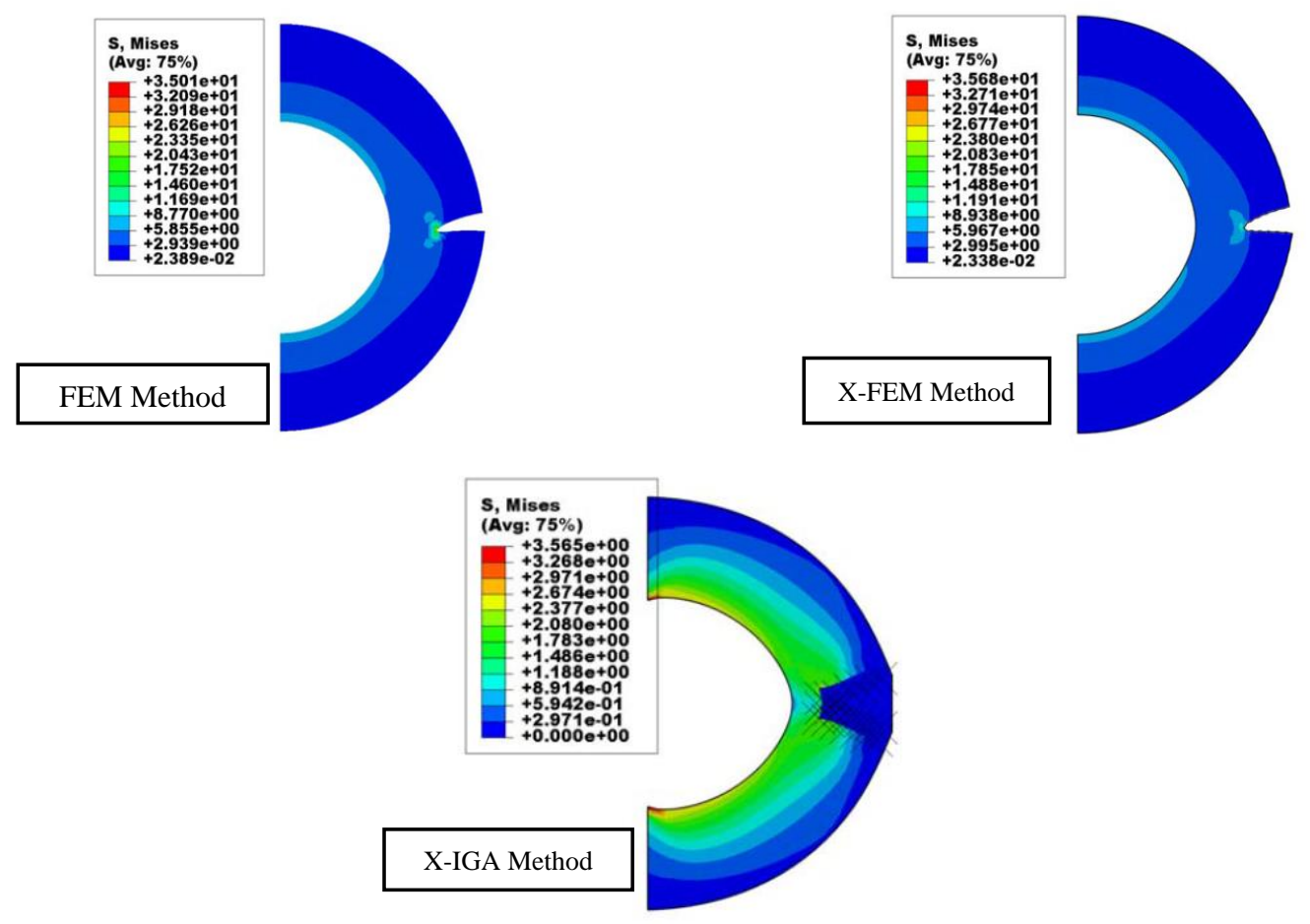

Fig. 7. The von Mises stress for the case of X-IGA, XFEM and FEM. 


\section{Conclusion}

In this paper, we presented the theoretical study extended isogeometric analysis XIGA. We also treated the formulation of the KI Constraint Intensity Factor for this new approach computed in the linear elastic domain in mode I, for the case of the half-tube with an elliptical crack. Then, we presented the steps of the implementation of the new X-IGA approach in the abaqus / standard software via Subroutine UEL. Finally, we carried out a comparative study between the new approach implemented and the other numerical methods (FEM, X-FEM) to study elliptic cracks.

The new X-IGA approach implemented under the Abaqus / Standard software has been successfully validated by corroborating its results against analytical and numerical computational models. The comparison of errors, shows that the calculation error of the KI factor using the new X-IGA approach is less compared to the other numerical methods. Therefore, We conclude that the use of the new X-IGA approach to study elliptic fissures has proved to be reliable in the study of cracked structures including in pipelines. The reliability generated by the proposed method can help solve the main problems of fracture mechanics and provide better accuracy at an economical cost.

\section{References}

[1] S. El Fakkoussi, H. Moustabchir, A. Elkhalfi, et C. I. Pruncu, « Application of the Extended Isogeometric Analysis (X-IGA) to Evaluate a Pipeline Structure Containing an External Crack », Journal of Engineering, vol. 2018, p. 1-10, oct. 2018.

[2] J. A. Cottrell, T. J. R. Hughes, et Y. Bazilevs, Isogeometric Analysis: Toward Integration of CAD and FEA. Wiley, 2009.

[3] A. Duval, T. Elguedj, H. Al-Akhras, et F. Maurin, « abqNURBS: implémentation d'éléments isogéométriques dans Abaqus et outils de pré-et post-traitement dédiés », in 12e Colloque national en calcul des structures, 2015.

[4] A. Stahl, T. Kvamsdal, et C. Schellewald, « Post-processing and visualization techniques for isogeometric analysis results », Computer Methods in Applied Mechanics and Engineering, vol. 316, p. 880-943, avr. 2017.

[5] F. Z. Li, C. F. Shih, et A. Needleman, « A comparison of methods for calculating energy release rates », Engineering Fracture Mechanics, vol. 21, nº 2, p. 405-421, 1985.

[6] N.-A. Noda, K. Oda, et K. Ishii, « Analysis of Stress Intensity Factors for Curved Cracks », JSME international journal. Ser. A, Mechanics and material engineering, vol. 37, nº 4, p. 360-365, 1994.

[7] H. Moustabchir, J. Arbaoui, Z. Azari, S. Hariri, et C. I. Pruncu, « Experimental/numerical investigation of mechanical behaviour of internally pressurized cylindrical shells with external longitudinal and circumferential semi-elliptical defects », Alexandria Engineering Journal, juin 2017 